\title{
Obstacle Detection and Avoidance Methods for Autonomous Mobile Robot
}

\author{
R. Gowtham \\ Junior Research Fellow, PSG College of Technology, Coimbatore, Tamil Nadu 641004, India
}

\begin{abstract}
In Recent Trends, Robotics plays a major role in manufacturing, mining, warfare, surgery, Education, Entertainment and other non-commercial application. Robots are very special since, a robot can be change based on the requirement and it's diligently obeying the order given by their masters. Secondly, the robot is induced to observe the real world and act upon and exhibit the task accurately to give perfect result at any moment. The feature of grasping the real world parameter to do a task in an iterative fashion leads to automated Robot. Generally, in thought a robot is moving object which react based on the feedback given to the system. Integrating the automatic and mobile behavior of a robot can be fulfilled only when the robot is capable of overcoming the obstacle and prove their maximum efficiency in find their absolute path towards destination. In this paper, we discuss about the sensor that help to feedback the system to detect obstacle and the actuator to avoid the obstacle.
\end{abstract}

Keywords: obstacle detection; obstacle avoidance; autonomous robot

\section{Introduction}

Utilization Robot are become popular due to their behavior and activity that the perform in the real world. ${ }^{[1]}$ There has been much recent activity toward achieving systems of multiple mobile robots engaged in collective behavior. Such systems are of interest for several reasons: tasks may be inherently too complex (or im-possible) for a single robot to accomplish, or performance benefits can be gained from using multiple robots; building and using several simple robots can be easier, cheaper, more flexible and more faulttolerant than having a single powerful robot for each separate task; and the constructive, synthetic approach inherent in cooperative mobile robotics can possibly yield insights into fundamental problems in the social sciences (organization theory, economics, cognitive psychology), and life sciences (theoretical biology, animal etiology). ${ }^{[5]}$ The complexity of real world environments is still a challenging problem. Uneven out-door environments, dynamic objects, cluttered scenes and the absence of simple features are known problems. Most navigation systems presented up to now use 2D sensors in combination with a 2D map for localization and path planning. First and foremost behavior of robot to move towards destination without collision with object on their path so here several possible method has been described to overcome those obstacles.

\section{Obstacle Detection}

Obstacle are the particles that oppose the robot to reach its destination in the shortest path or the objects that are surrounded by the robot incase the robot is random towards the destination. There are several methods to detect the obstacles based on the sensor which is used. ${ }^{[2]}$ Path planning for autonomous vehicles requires that the map of all potential obstacles be produced in real time using available sensor data. For Indoor it is easy to design a map and feed into robot to facilitate about the permanent obstacles. ${ }^{[3]}$ Sensors are the sensing organs of technical systems. They collect information about variables in the environment as well as on non-electrical system parameters. They provide the results as electrical signals. Sensors are an essential part of power generation and distribution systems, automated industrial processes, traffic management systems, as well as environmental and health maintenance systems.

\section{Ultrasonic Sensor}

Ultrasonic transducers which are referred as ultrasonic sensor that convert audio to electrical signal and/or electrical signal to audio; hence a pair of sensor is used to non contact distance measurement. A high frequency sound wave is generated which is called as signal and it is reflecting by the object which is called as echo the interval between the signal and echo will give the distance from the object similar to the mechanism used in sonar's and radar's. The values obtain between $5 \mathrm{v}$ to $0 \mathrm{v}$ calibrated across $450 \mathrm{~cm}$ to $0 \mathrm{~cm}$ based on the range of ultrasonic echoes.

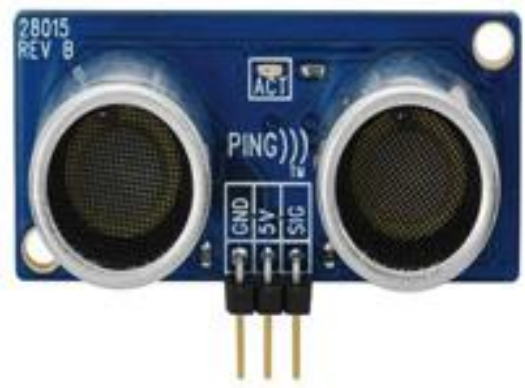

Figure 1: Ultrasonic sensor

The super close value will be $2 \mathrm{~cm}$ below this limit the angle of reflected echo will be zero since the reflected angle is 15 degree. [4]The Parallax PING))) ${ }^{\mathrm{TM}}$ ultrasonic distance sensor provides precise, non-contact distance measurements from about $2 \mathrm{~cm}$ ( 0.8 inches) to 3 meters ( 3.3 yards). It is very easy to connect to microcontrollers such as the BASIC Stamp, Propeller chip, or Arduino, requiring only one I/O pin. The ultrasonic sensor has three pin pin1-ground, pin2supply $5 \mathrm{v}$, pin3-signal.The output is observed in pin-3 in corresponding response of echoed signal.

\section{Infrared Sensor}

Infrared sensor is combination of an infrared light source (LED) and photodiode. Infrared sensor are low cost and low 


\section{International Journal of Science and Research (IJSR) \\ ISSN (Online): 2319-7064 \\ Index Copernicus Value (2013): 6.14 | Impact Factor (2014): 5.611}

power consumption that the other light sensor. The range of detection will be less than $15 \mathrm{~cm}$ with $2 \mathrm{v}$ dc supply. The sensitivity of infrared is better to find the object even it is moving so infrared sensor plays a vital role in short range obstacle detection. The scattering of the light becomes more as the object moves away from the robot, at a point the reflected rays at photodiode will become zero. Three pins for vec 5v de supply ground and signal. A single PIR detector can only output two different states and cannot be used to measure distances between the radiation object and detector. In order to estimate the position of an infra red radiation object, the problem of the location of object state space is down to Multi-degree of freedom FOV division in this paper. At first a radial distance modulated method is proposed, therefore the distance between object and detector can be measured by several PIR detectors in a single sensor node, and then with several sensor nodes data fusion the position of the object can be estimated.

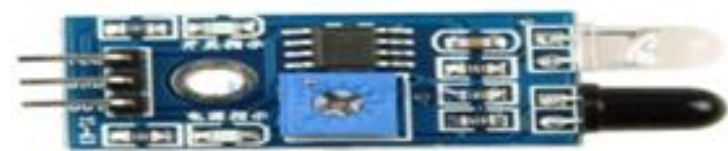

Figure 2: IR Sensor

\section{Proximity Sensor}

Proximity sensor enables a robot to detect the object either moving or stable. The sensor detects the range by finding the magnetic flux beam deflection by the object which is sense by the coil that generated appropriate voltage difference at the end. Proximity sensor is highly stimulus to the moving object in near field since the range of detection is limit to few centimeters however today long range proximity sensor is come into existence in detection up to few meter. This sensor are reliable since there is no any mechanical part and long life since there are not directly contact with any object physically.

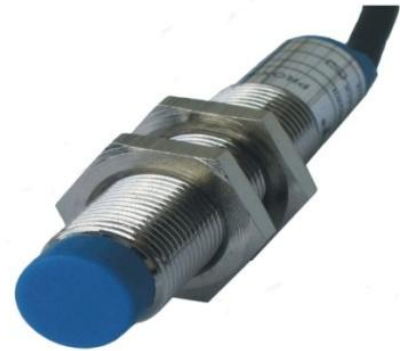

Figure 3: Proximity Sensor

\section{Tilt Sensor}

Tilt sensor is comprised of two ball inside a closed metal tube the ball at top move according to the movement of the robot and the ball at the bottom is pushed to make the contact to be connected which acts like s switch. In robot this sensor are used at the edges of the robot which bends when the robot touch any physical object.

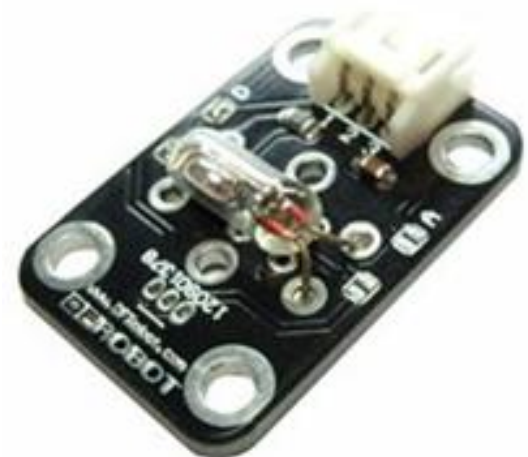

Figure 4: Tilt Sensor

\section{Push Sensor}

Spring sensor are similar to tilt sensor it perform like a switch as it touch any object but little stress is need between the robot and the object in order to press the spring to make contact to the terminal. These sensors are less reliable due to physical movement and calibration is often needed due to stress and strain of the spring.

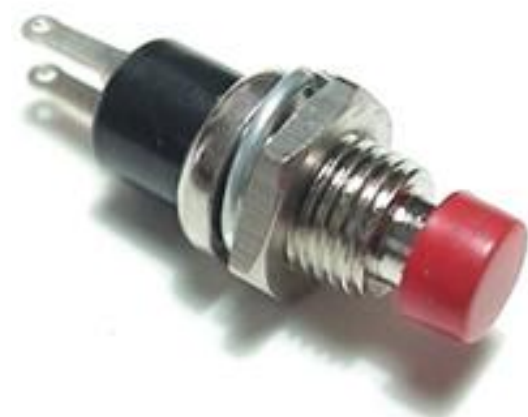

Figure 5: Push Switch

\section{Accelerometer}

Accelerometer is sensitivity to sudden change in the orientation of the robot. When robot hit an object the accelerometer mark a motion in opposite direction according to Newton's third law. From the changes in the accelerometer a dc voltage is feedback to the robot. Accelerometer circuit shown in figure 6 .

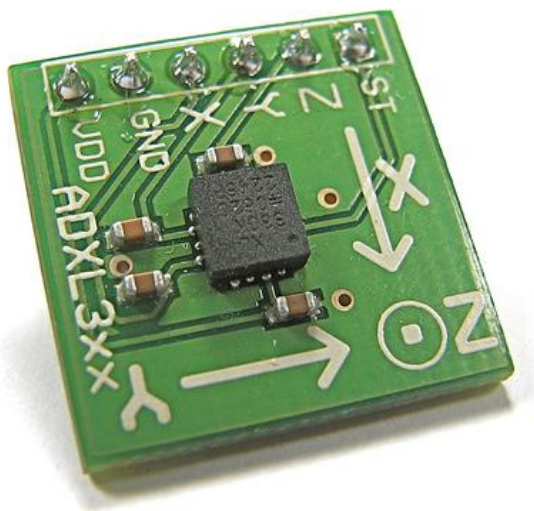

Figure 6: Accelerometer

\section{Gyroscope}

Gyroscope is sensitivity to continuous change in the orientation of the robot. When the robot hit by an obstacle the large variation is marked as detection of obstacle. Both gyroscope and accelerometer can detect obstacle in three dimensions as shown in figure 7. 


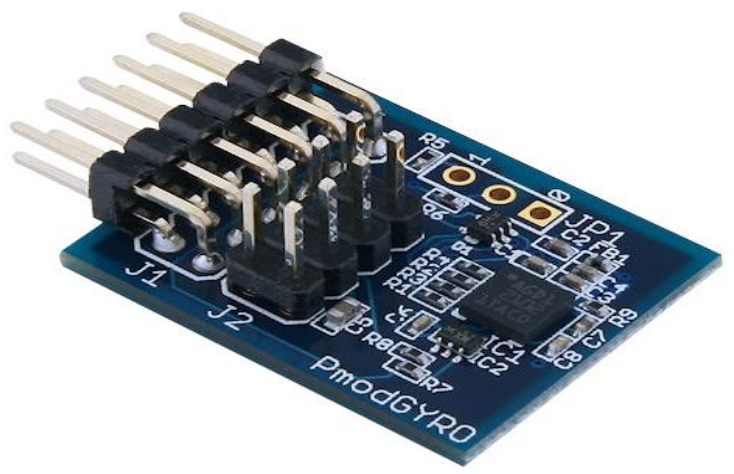

Figure 7: Gyroscope

\section{Obstacles Avoidance}

The sensor can be used to avoid collision of robot with any obstacles that is present in the path of robot. Based on the detection the system is classified as two type's a. Direct detection and avoidance $\mathrm{b}$. Indirect detection and avoidance.

\section{Direct detection and avoidance (DDA)}

In this method, the robot is touch or colloid few second and as a response it moves away from the obstacle which means the robot is in physical contact with object and respond accordingly.

DDA includes sensor like tilt sensor, push switch, spring sensor, accelerometer and gyroscope where there response to robot is created only after the robot is in contact with obstacles. For push switch the robot is build and the outer edges of the robot are attached with push switch. Moreover these push switch should be fixed and reliable so that the stimulus will be perfect.

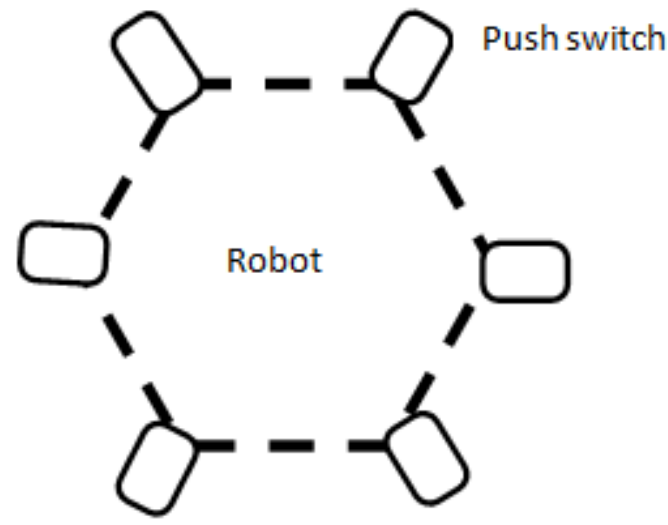

Figure 8: Push switch DDA model

The same approach can be achieved through Accelerometer where the stimulus is appeared as opposite force. Care take while determination of state changes in accelerometer since the response generated on opposing side.

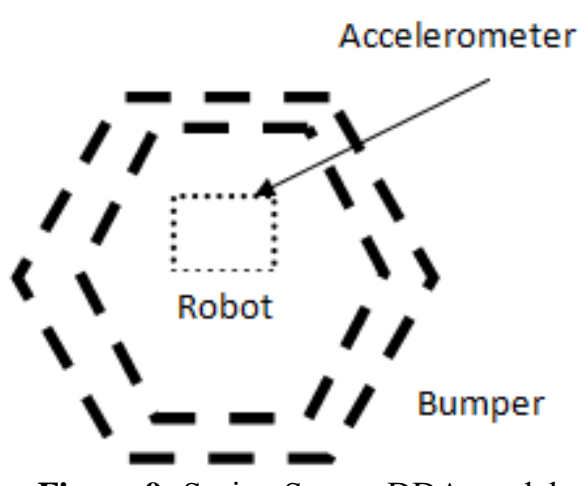

Figure 9: Spring Sensor DDA model

The same approach can be achieved through gyroscope where the stimulus is appeared as force continuously varies in same direction as that of robot. The difference in axis is measured and if those difference are large then it is account as it hit the obstacle so, the robot started moving in opposite direction.

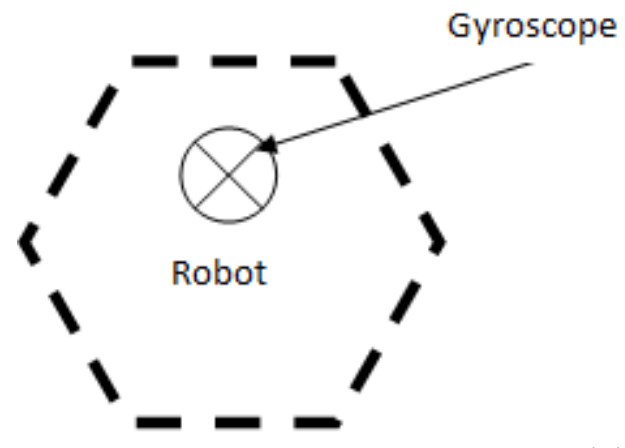

Figure 10: Gyroscope Sensor DDA model

Tilt sensor is made as a flexible finger placed at the edge of the robot while the finger bends the tilt sensor rise up and make the contact to generate the response signal.

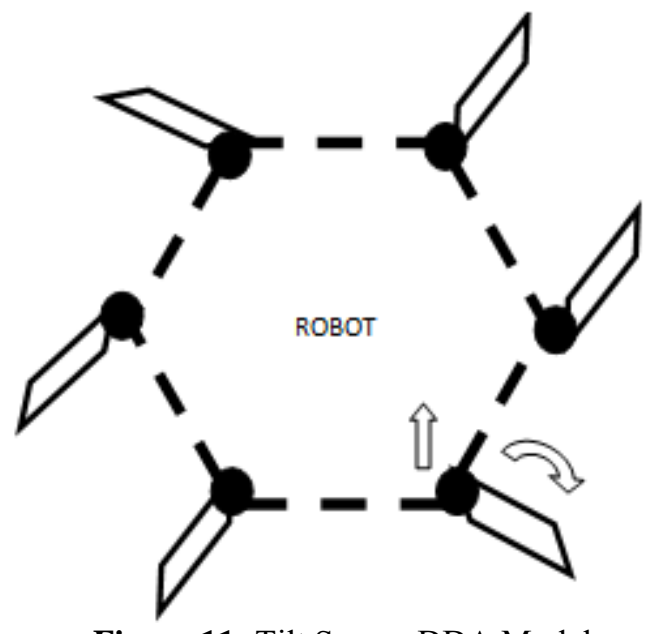

Figure 11: Tilt Sensor DDA Model

\section{Indirect Detection and Avoidance (IDA)}

In this method, the robot is not touch or colloid with the obstacles it moves away from the obstacle due to the response received through light or sound enable the robot to find its own path without any physical contact with the object around them. IDA method becomes common in obstacle detection for robot because of their reliable and range of detection. Sensors like ultrasonic, infrared, 


\section{International Journal of Science and Research (IJSR) \\ ISSN (Online): 2319-7064}

Index Copernicus Value (2013): 6.14 | Impact Factor (2014): 5.611

proximity are quiet easy and cheap for this types of applications moreover the response is quick comparative to DDA method.

Ultrasonic sensor should be fixed at each face of the robot since the cover a wide angle they can be fixed in the face in spite of their edges. Ultrasonic transceiver pair at each face detect the object as it move at locate it immediate free position and move by determining an non obstacle path at every instance.

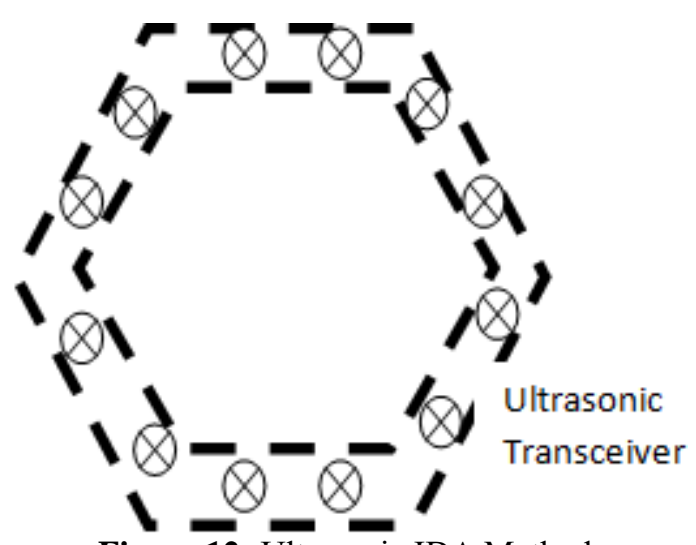

Figure 12: Ultrasonic IDA Method

IR sensor should be fixed at each face edge of the robot since the IR sensor cover small portion due to the scattering of light so edge will perfect to detect the obstacle. In case of square type chassis the IR sensor should be placed in middle to avoid number of sensor while considering at the edges.

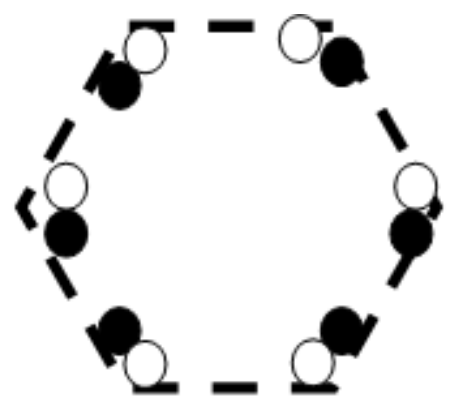

Figure 13: IR Sensor IDA Method

Proximity sensor can detect the change in their magnetic field thus the object which move by blocking their magnetic field will generate a stimulus in response to the object no matter whether the object moves or the robot move the magnetic field should be cut be either moving object or moving robot. Whatever the regular chassis may be it should fixed at the middle.

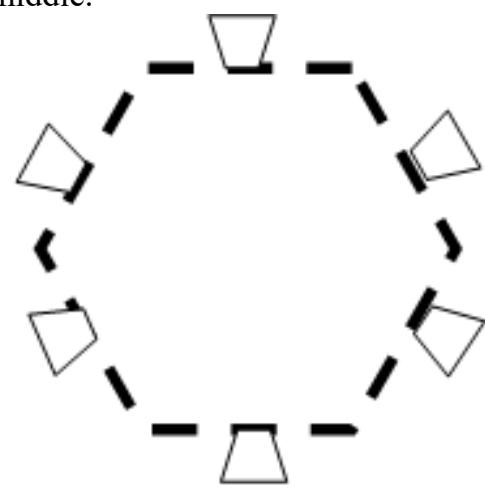

Figure 14: Proximity Sensor

\section{Conclusion}

In this paper, the sensor which are used for obstacle detection are analyzed and also discussed on the method of detection of the obstacles with illustration and projected where to place the sensor in the robot to obtain excellent result of the sensor. Future more, the use of sensor is also discussed with their mechanism and explains how suitably they can be attached with the autonomous robot.

\section{Future Work}

In future, the sensor which is described in the above are planned to utilize to develop an auto IPS (Indoor Positioning System) using indoor mapping technology. A wireless network manager use to point the location so that the robot moves to the destination in a shortest path and also avoiding all the obstacles coming across which can be monitored and controlled through the network manager.

\section{References}

[1] Y. Uny Cao, Alex S. Fukunaga, Andrew B. Kahng, 1997 "Cooperative Mobile Robotics: Antecedents and Directions", Computer Science Department, University of California, Los Angeles \& Propulsion Laboratory, California Institute of Technology, Pasadena, Kluwer Academic Publishers, Boston, Autonomous Robots, 4, 1-23 (1997).

[2] R. Manduchi, A. Castano, A. Talukder and L. Matthies,2005 , "Obstacle Detection and Terrain Classification for Autonomous Off-Road Navigation" University of California at Santa Cruz, Santa Cruz \& Jet Propulsion Laboratory, California Institute of Technology, Pasadena, Springer Science + Business Media, Inc, Autonomous Robots 18, 81-102.

[3] Baihua Shen, Guoli Wang, 2013, School of Information Science \& Technology, Sun Yat-Sen University,School of Information Engineering, Guangdong University of Technology, "Distributed Target Localization And Tracking With Wireless Pyroelectric Sensor Networks", International Journal On Smart Sensing And Intelligent Systems vol. 6.

[4] Parallax ping ultrasonic sensor \#28105 version v2.0 2/4/2013

[5] Pandey, A. ; Mech. Eng. Dept., Nat. Inst. of Technol., Rourkela, India ; Sonkar, R.K. ; Pandey, K.K. ; Parhi, D.R.2014, "Path planning navigation of mobile robot with obstacles avoidance using fuzzy logic controller", Intelligent Systems and Control (ISCO), 2014 IEEE 8th International Conference.

[6] Valentin Magori,1994 "Ultrasonic Sensors in Air Corporate Research and Development", Siemens AG. Munich, Germany.

[7] Son Duongand Min-Hyung Choi, 2013 Department of Computer Science and Engineering, University of Colorado Denver, "interactive full-body motion capture using infrared sensor network" International Journal of Computer Graphics \& Animation (IJCGA) Vol.3, No.4.

[8] Budiharto, W. ; Sch. of Comput. Sci., Binus Univ., Jakarta, Indonesia ; Rachmawati, R.N. 2012, "Moving Obstacles Avoidance for Humanoid Service Robot 


\section{International Journal of Science and Research (IJSR) \\ ISSN (Online): 2319-7064}

Index Copernicus Value (2013): 6.14 | Impact Factor (2014): 5.611

Using Stereo Vision and Bayesian Approach", Modelling Symposium (AMS), 2012 Sixth Asia IEEE.

[9] Lin Xiaolong ; Sch. of Mech. Eng. \& Autom., Northeastern Univ., Shenyang, China ; Wang Haiyang; Wei Yangtao ; Duan Jinchao, 2015 "Design of a wireless obstacle-crossable exploration robot" , Control and Decision Conference (CCDC), 2015 27th Chinese IEEE.

[10] Chun-Han Lin ; Dept. of Comput. Sci., Nat. Tsing Hua Univ., Hsinchu, Taiwan ; Chung-Ta King, 2010, "Sensor-Deployment Strategies for Indoor Robot Navigation", Systems, Man and Cybernetics, Part A: Systems and Humans, IEEE Transactions on Volume:40, Issue: 2. 